Tohoku J. exp. Med., 1983, 141, Suppl., 439-445

\title{
The Prevalence of Neuropathic Symptoms in Diabetic Patients Newly Referred to Our Hospital
}

\author{
Yoshiatsu Takahashi, Yukiko Inoue and Yukimasa \\ Hirata \\ Diabetes Center, Tokyo Women's Medical College \\ Kawada-Cho, Shinjuku-ku, Tokyo 162, Japan
}

\begin{abstract}
Takahashi, Y., Inoun, Y. and Hrrata, Y. The Prevalence of Neuropathic Symptoms in Diabetic Patients Newly Referred to Our Hospital. Tohoku J. exp. Med., 1983, 141, Suppl., 439-445 — 100 diabetic patients who were newly referred to our hospital and 50 normal controls received 1) physical and chemical examinations, 2) a self-rating deperession score (SDS), and 3) an examination consisting of 18 questions to detect subjective neuropathic symptoms. Many more neuropathic symptoms were found in diabetics than in normal controls; these symptoms included pain and numbness of the extremities, cold sensation, dizziness, constipation, impotence and sweating disorders. Few patients with neuropathic symptoms, however, exhibited neurological findings (tendon reflex, sensory disturbance or dorsal pulsation) or blood glucose control measured by $\mathrm{HbA}_{1}$. The likelihood of symptoms in the feet or in the urogenital tract increased with the duration of diabetes mellitus. Diabetics were significantly more depressive than normal controls shown by the result of SDS. - _ neuropathic symptom; diabetes mellitus; $\mathrm{HbA}_{1}$; SDS
\end{abstract}

Although diabetic neuropathy is probaly the most common complication of diabetes, it remains the least understood and the least explored (Ellenberg 1977). Many papers about the incidence of diabetic neuropathy have been writtea but estimates of the incidence vary widely (0 to $93 \%$ ) (Bruyu and Garland 1970), according to the criteria employed for diagnosis. Few reports make comparisons between diabetics and normal controls whose sex and age match those of the diabetics. The purpose of this report is to find the prevalence of neuropathic symptoms of diabetics in comparison to normal controls and to evaluate those symptoms objectively by $\mathrm{HbA}_{1}$, neurological findings and psychological tests.

\section{Subjects and Methods}

Included in this study were 100 ambulatory diabetic patients, newly referred to our hospital during the course of this study, and $\mathbf{5 0}$ normal controls whose sex and age were matched with the diabetics (Table 1). The diagnosis of diabetes mellitus was made by our colleagues by studying the metabolisn and clincal history of the subjects.

Table 2 shows the clinical characteristics of 100 diabetics who received routine physical and chemical examinations. $\mathrm{HbA}_{1}$ was measured as an indication of blood glucose control 
during the preceding 6-week period; the normal value comprises 5.5 to $8.5 \%$ of total hemoglobin. All subjects received the examination, consisting of 18 questions, for subjective symptoms concerning the diabetic neuropathy. All subjects received a test of the selfrating depression score (SDS), developed by Zung (1965) and consisting of a 20-item scale for rating a variety of symptoms associated with depression.

Student $t$-test and the $\chi^{2}$ test were used in statistical comparison.

Table 1. Subjects in the study

\begin{tabular}{llc} 
& Diabetics & Normal controls \\
\hline Number & 100 & 50 \\
Age (Mean \pm s.d. years) & $50 \pm 11$ & $46 \pm 10$ \\
Sex (Male/Female) & $70 / 30$ & $33 / 17$
\end{tabular}

Age and sex do not differ between diabetics and controls.

TABLE 2. Clinical characteristics of 100 diabetics

\begin{tabular}{clllcr}
\hline & Characteristics & $\%$ & \multicolumn{2}{c}{ Characteristics } & $\%$ \\
\hline Decreased & PTR & 38 & $\mathrm{HbA}_{1}(\%)$ & $<10$ & 58 \\
or absent & ATR & 48 & & $10 \sim 12$ & 24 \\
Retinopathy & negative & 64 & & $12<$ & 18 \\
& positive (Scott I, II) & 22 & Duration & $<1$ & 33 \\
& positive (Scott III - ) & 14 & of DM & $1 \sim 5$ & 27 \\
Proteinuria & negative & 87 & (years) & $5 \sim 10$ & 24 \\
& positive & 13 & & $10<$ & 16 \\
\hline
\end{tabular}

PTR, patellar tendon reflex; ATR, Accilles tendon reflex.

\section{RESULtS}

Fig. 1 represents the prevalence of neuropathic symptoms in diabetics compared with normal controls. The prevalence of numbness of the extremities, ptotic sensation, constipation, reduced force of urination, defect of orgasm and sweating disorders, was significantly higher in diabetics than in normal controls $(p<0.005)$. The prevalence of such symptoms as a hot sensation of extremties, pain in the calf on walking, alternation of diarrhea and constipation, diarrhea, awareness of residual urine and decreased libido, did not differ significantly between diabetics and normal controls. Although the symptom of decreased libido, in particular, occurred most frequently in the answers to the 18 questions, there was no difference in prevalence when compared with those patients whose sex and age matched normal controls.

The proportion of abnormal Achilles tendon reflexes (ATR) among each group of diabetics showing neuropathic symptoms was similar to the percentage of abnormal ATR in the 100 diabetic patients. (Fig. 2, Table 2) 


Pain in extremities
Numbness of extremities
Cold sensation of extremities
Hot sensation of extremities
Pain in the calf on walking
Push oneself up at standing
Difficulty going up and down stairs
Double vision, ptotic sensation
Dizziness or light-headedness
Alternation of diarrhea and
constipation
Diarrhea
Constipation
Reduced force of urination
Awareness of residual urine
Decreased libido
Erectile impotence
Defect of orgasm
Sweating disorders

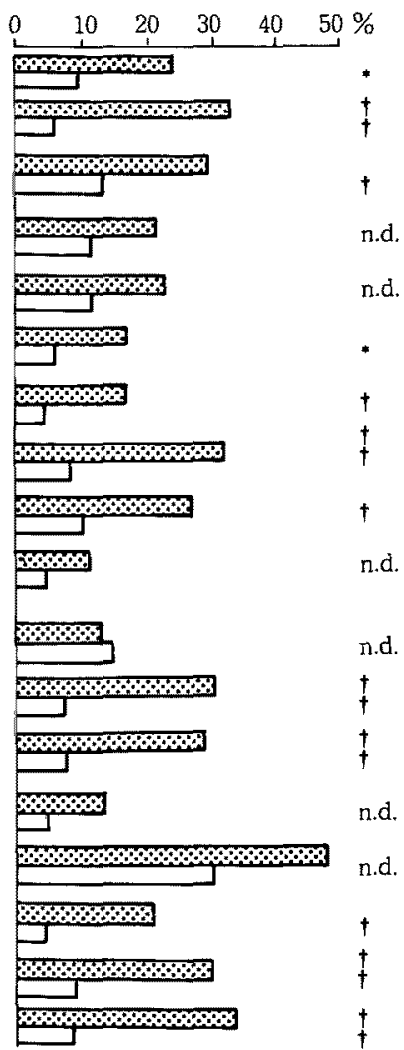

Fig. 1. The prevalence of neuropathic symptoms in diabetics (3) and normal controls $(\square)$.

${ }^{*} p<0.1, \dagger p<0.05, \ddagger p<0.005$, n.d. $=$ not different. The $\chi^{2}$ test was used in statistical comparison.

The distribution of blood glucose control in each group of diabetics with neuropathic symptoms was also nearly the same as that in the 100 diabetics (Fig. 3 , Table 2). Therefore, there was found to be little relation between diabetic patients with neuropathic symptoms on the one hand, and neurological findings and blood glucose control on the other.

Fig. 4 shows the duration of diabetes mellitus in each patient in relation to the neuropathic symptoms. Foot symptoms (pain, numbness, cold sensation in extremities) and urogenital symptoms (awareness of residual urine, decreased libido, erectile impotence and defect of orgasm) seemed to be related to the duration of diabetes mellitus.

Fig. 5 shows the results of the SDS, for which a score under 40 is considered normal, and a score over 50 indicats depression (Ikemi 1974). The diabetics scored $35.2 \pm 9.8$ and controls $30.8 \pm 7.2$, the difference being significant by $t$-test comparison $(p<0.025)$. On the other hand, there was no difference between the 
Pain in extremities

Numbness of extremities

Cold sensation in extremities

Hot sensation in extremities

Pain in the calf on walking

Push oneself up at standing

Difficulty going up and down stairs

Double vision or ptotic sensation

Dizziness or light-headedness

Alternation of diarrhea and constipation

Diarrhea

Constipation

Reduced force of urination

Awareness of residual urine

Decreased libido

Erectile impotence

Defect of orgasm

Sweating disorders

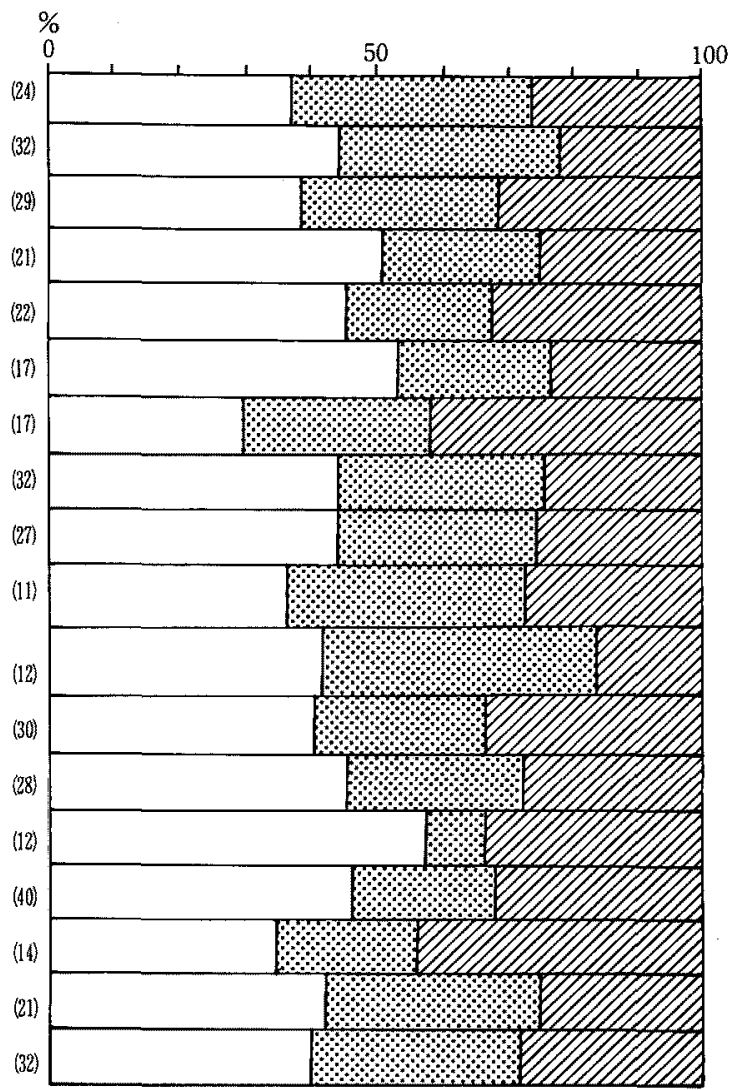

Fig. 2. The proportion of abnormality of Achilees tendon reflex among the diabetics showing each neuropathic symptom.

$\square$, normal ; 7 , decreased; $\square \mathbb{Z}$, absent. $(\quad)$ is total number of the subjects.

scores for positive and negative symptoms, such as pain in the extremities, numbness in the extremities, decreased libido, erectile impotence or defect of orgasm. The score for positive sweating disorders was $39.6 \pm 9.6$ and the score for negative disorders was $32.6 \pm 9.3$, which was significantly different $(p<0.005)$.

\section{Discussion and Conclusion}

Since symptoms of pain in the extremities were not only confined to peripheral neuropathy, but also included cases of arthralgia and shoulder stiffness, the prevalence of these symptoms was thought not to differ significantly to that seen in normal controls. Likewise, diarrhea, decreased libido and pain in the calf on walking are not uncommon symptoms in middle aged people. Therefore, the prevalence of these symptoms did not differ between diabetics and normal controls.

In diabetics, neuropathy has long been thought of as a result of a prolonged 
Pain in extremities

Numbness of extremities

Cold sensation in extremities

Hot sensation in extremities

Pain in the calf on walking

Push oneself up at standing

Difficulty going up and down stairs

Double vision, ptotic sensation

Dizziness or light-headedness

Alternation of diarrhea and constipation

Diarrhea

Constipation

Reduced force of urination

Awareness of residual urine

Decreased libido

Erectile impotence

Defect of orgasm

Sweating disorders

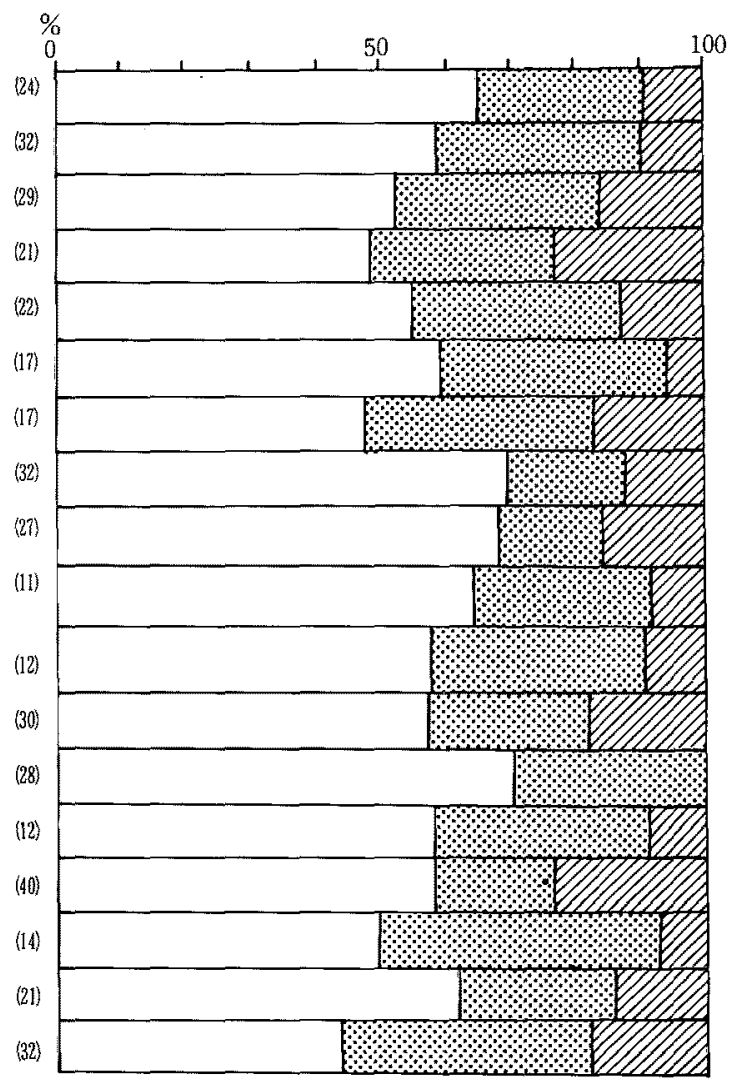

Fig. 3. Distribution of blood glucose control of the diabetics showing each neuropathic symptom.

$\square$, good control $\left(\mathrm{HbA}_{1}<10 \%\right)$; fair control $\left(\mathrm{HbA}_{1}=10 \sim 12 \%\right)$; $\mathbb{Z} \mathbb{Z}$, poor control $\left(\mathrm{HbA}_{1}>12 \%\right)$. ( ) is total number of the subjects.

period of poor diabetic control. However, the occasional lack of any relation between the duration or severity of abnormal glucose metabolism and the presence of neuropathy underscores the possibility that diabetic neuropathy is a concomitant rather than a complication (Ellenberg 1963). This study shows that almost all neuropathic symptoms had little relation to neurological findings and blood glucose control. Some neuropathic symptoms were strongly related to the duration of the diabetes. However, these results might be overshadowed by an aging factor and it must not be forgotten that the precise duration of diabetes mellitus in the adult cannot be measured.

It has been generally considered that diabetes has a tendency to be depressive, and this study confirmed this fact by SDS. However, there was no difference in the scores of subjects with positive and negative neuropathic symptoms except for one symptom. This probably means that diabetes essentially has a depressive tendency whether the symptoms are positive or not. 
Pain in extremities

Numbness of extremities

Cold sensation in extremities

Hot sensation in extremities

Pain in the calf on walking

Push oneself up at standing

Difficulty going up and down stairs

Double vision or ptotic sensation

Dizziness or light-headedness

Alternation of diarrhea and constipation

Diarrhea

Constipation

Reduced force of urination

Awareness of residual urine

Decreased libido

Erectile impotence

Defect of orgasm

Sweating disorders

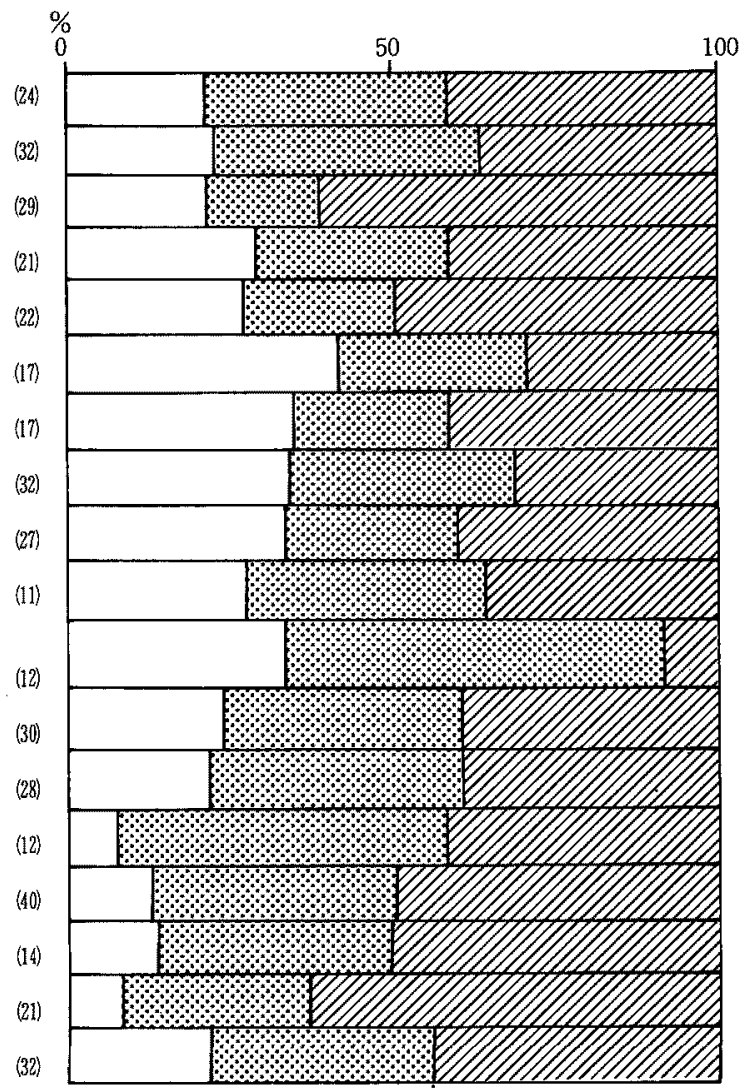

Fig. 4. Duration of diabetes mellitus in subjects with each neuropathic symptom. , 0 1 year; 3 , 1 5 year ; $\mathbb{Z}, 5 \sim$ year, ( ) is total number of the subjects.

In conclusion, the nouropathic symptoms in diabetics could not always be related to neuropathy and metabolic control. Roughly about one half of subjective symptoms seemed to be related to neuropathy, whereas the other half were not. 
Diabetic patients

Normal controls
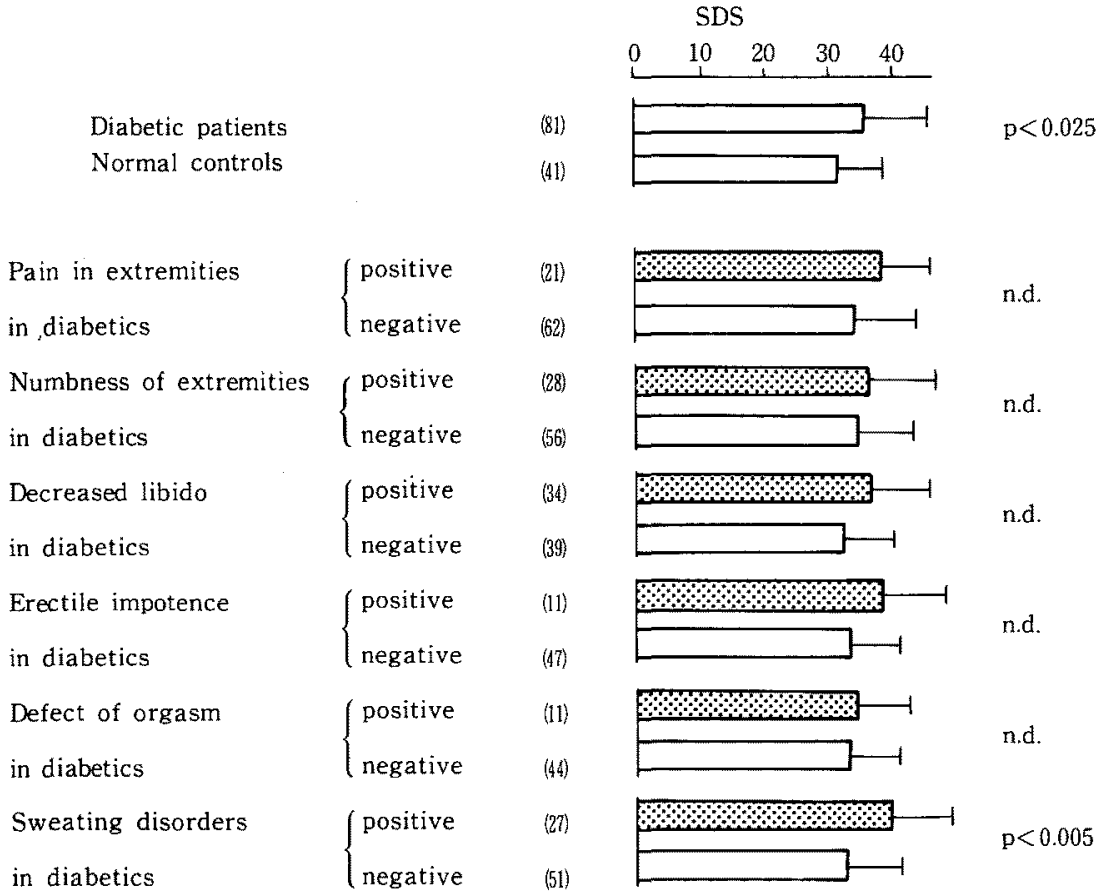

Fig. 5. Results of SDS in several analyses (mean \pm S.D.). ( ) is the total number of the subjects.

Student's $t$-test was used in statistical comparison.

\section{References}

1) Bruyn, G.W. \& Garland, H. (1970) Neuropathies of endocrine origin. In : Handbook of Neurology. edited by P.J. Vinken \& G.W. Bruyn, North Horlland Publishing Co., Amsterdam, p. 29.

2) Ellenberg, M. (1963) Diabetic complications without manifest diabetes. J. Amer. med. Ass., 183, 118-122.

3) Ellenberg, M. (1977) Diabetic neuropathy; Present and future trends. In: Diabetes Proceedings of the $I x$ Congress of the International Diabetes Federation, edited by J.S. Bujaj, Excerpta Medica, Amsterdam, pp. 643-648.

4) Ikemi, Y. (1974) In: Modern Psychosomatic Medicine. lst ed., Ishiyaku Press, Tokyo, p. 178.

5) Zung, W.W.K. (1965) Self-rating depression scale in an outpatient clinic. Arch. gen. Psychiat., 13, 508. 